\title{
La evolución de las creencias de los profesores de Inglés en servicio sobre la enseñanza de la gramática
}

\author{
M.Sc. Julio César Roa Rocha \\ Docente investigador \\ UNAN-Managua, FAREM-Matagalpa \\ juliorn27@yahoo.com
}

Fecha de recepción: 09 de octubre, 2019 / Fecha de aceptación: 23 de octubre, 2019

https://doi.org/10.5377/torreon.v8i21.8853

Palabras clave: docentes en servicio, creencias, influencia, docentes en servicio, formación docente.

\section{RESUMEN}

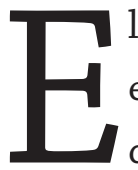

1 propósito de este estudio fue medir cómo cambian las creencias sobre la enseñanza de la gramática de los maestros de inglés en servicio en Nicaragua después de completar un programa de pregrado de enseñanza de inglés de 5

años y con cierta experiencia de enseñanza acumulativa. Se recopilaron datos de cuestionarios, diarios y observaciones de la práctica en el aula. Los participantes fueron 20 profesores de inglés en la escuela secundaria en servicio que estaban tomando un programa de capacitación de un año y fueron seleccionados mediante muestreo de conveniencia. Los resultados mostraron que había inconsistencia entre las creencias de los maestros y la práctica en el aula y sus creencias están influenciadas de alguna manera por sus experiencias como estudiantes y maestros. En conclusión, las creencias de los maestros son a menudo un reflejo de cómo ellos mismos fueron enseñados y sus experiencias, ya sea como maestros o estudiantes, se reflejan en su práctica docente. 


\section{INTRODUCCIÓN}

La enseñanza es un proceso rodeado de decisiones que toman fundamentalmente. Los maestros tienen que tomar decisiones sobre cómo enseñar y evaluar sus lecciones desde el principio hasta el final. La mayoría de las decisiones que toman los maestros son vitales para el proceso de aprendizaje. Dentro del campo de la investigación educativa contemporánea, y especialmente dentro del campo de la enseñanza de lenguas extranjeras y segundas lenguas, la cognición docente se ha convertido en un área de gran interés en los últimos treinta años (Borg 2003).

De hecho, ahora se sostiene ampliamente que comprender lo que los maestros piensan y creen es esencial para comprender (y por lo tanto afectar) lo que sucede en el aula (Borg 2005). No obstante, los programas de formación docente tradicionalmente han descuidado tener en cuenta las creencias y experiencias de enseñanza y aprendizaje que los alumnos docentes traen consigo. Aunque se han utilizado muchos términos diferentes, como conocimiento pedagógico, teorías de práctica, creencias, teorías personales, etc., los investigadores se han interesado en comprender lo que los maestros piensan, saben y creen sobre la enseñanza y el aprendizaje de idiomas (Borg 2003). Este interés surge de la idea generalmente aceptada de que las creencias de los docentes influyen en sus percepciones y decisiones y, por lo tanto, deben entenderse para mejorar las prácticas docentes y los programas de capacitación docente (Farrell 2005). Además, la teoría constructivista en la enseñanza afirma que los maestros construyen estas creencias en gran medida a través de su experiencia como estudiantes de idiomas y que estas creencias a menudo son resistentes al cambio (Richards, Gallo y Renandya 2001).

El propósito de este estudio fue medir los cambios de las creencias de los maestros en servicio sobre la enseñanza de la gramática que traen consigo después de completar un programa de pregrado de cinco años y con cierta experiencia acumulada, para ver si y como sus creencias se ven afectadas como estudiantes y profesores de idiomas. Para el propósito de este estudio, se recopilaron datos administrando cuestionarios diarios y observación en el aula. Los participantes fueron 20 maestros de inglés en servicio que fueron seleccionados mediante muestreo de conveniencia. Finalmente, los datos se analizaron utilizando métodos cualitativos y cuantitativos. Los resultados mostraron que existe una contradicción con las creencias de los maestros en servicio sobre la enseñanza de la gramática y cuál es su práctica en el aula. En conclusión, la mayoría de lo que hacen los maestros en servicio en el aula es solo un reflejo de cómo se les enseñó, todos los maestros fueron estudiantes una vez.

\section{REVISIÓN LITERARIA}

En su revisión de la investigación de la enseñanza de la gramática, Borg (1999) señaló la falta general de consenso obtenida de muchos años de investigación de "mejores prácticas" 
en el área de la enseñanza de la gramática de un segundo idioma. Citando el cambio en la investigación de la enseñanza de idiomas hacia un énfasis en la cognición del maestro, Borg enfatizó la importancia de explorar las creencias del maestro sobre la enseñanza de la gramática para comprender mejor cómo se toman las decisiones y, por lo tanto, mejorar la educación del maestro L2.

La afirmación de Borg fue confirmada por un estudio sobre las creencias centrales de los maestros realizado por Richards, Gallo y Renandya (2001), en el que se encuestó a 126 maestros de inglés en servicio sobre sus creencias centrales sobre la enseñanza. Después de clasificar las respuestas, se encontró que la mayoría de las respuestas se centraron en la enseñanza de la gramática. Esto sugiere que las creencias sobre la enseñanza de la gramática pueden estar entre las menos propensas a cambiar, ya que se identificaron como creencias centrales, que a menudo están vinculadas a sentimientos fuertes y están muy arraigadas. También significa que es importante saber qué creencias aportan los futuros docentes a su formación profesional, si dicha formación pretende provocar algún tipo de cambio en esos docentes.

Hay muchos estudios que respaldan los hallazgos de Richards, Gallo y Renandya (2001). Borg (2003) citó muchos estudios (Eisenstein-Ebsworth y Schweers 1997; Burgess y Etherington 2002; Brumfit, Mitchell y Hooper 1996; Mitchell, Brumfit y Hooper 1994a, 1994b; Mitchell y Hooper 1992) que exploraron las opiniones de los maestros sobre la instrucción dela gramática. Todos estos estudios encontraron que los maestros estaban predispuestos a enseñar gramática de la manera en que se les enseñó; en la mayoría de los casos de manera explícita, basada en oraciones. Las teorías de la enseñanza de idiomas basadas en SLA parecían tener poco efecto en cambiar la forma en que estos maestros abordaban la enseñanza de la gramática en sus clases.

Otro estudio importante en el área de las creencias sobre la enseñanza de la gramática es el de Andrews (2006), en el que examinó la evolución de la Conciencia del Idioma del Maestro (TLA) en relación con la gramática de tres profesores de inglés con experiencia. Estos maestros habían participado en un estudio previo sobre TLA (Teacher Language Awareness) en sus primeros años de enseñanza. Curiosamente, Andrews descubrió que el conocimiento de los maestros sobre la gramática y sus creencias sobre la enseñanza de la gramática no cambiaron en gran medida después de 10 años de práctica profesional.

De la investigación mencionada aquí, se puede concluir que las creencias de los maestros sobre la enseñanza de la gramática se encuentran entre las más difíciles de cambiar. Esto podría deberse a que los primeros encuentros de la mayoría de los maestros con la enseñanza de la gramática suceden cuando están en la escuela secundaria y, por lo tanto, quedan muy impresos en sus mentes mucho antes de llegar a un programa de capacitación docente. En consecuencia, 
los programas de capacitación que no tienen en cuenta las experiencias y creencias previas de los docentes en formación o en servicio tienen poco impacto.

\section{Investigación sobre las creencias de los docentes en los programas de formación docente.}

Se han realizado muchas investigaciones sobre el efecto de la formación de docentes en la cognición docente en el campo de la enseñanza de lenguas extranjeras y de segunda lengua. Una revisión muy citada sobre este tema es Kagan (1992), cuyos resultados mostraron que la formación del profesorado tuvo un impacto insignificante en las creencias de los docentes (según lo citado por Borg 2003). Aunque los estudios posteriores han refutado sus ideas, la naturaleza exacta por la cual la formación del profesorado influye en las creencias de los docentes aún no está clara. Sin embargo, estudios más recientes han arrojado luz sobre lo que sucede con las creencias de los maestros en los programas de capacitación.

Uno de estos estudios es el de Freeman (1993), que fue un estudio longitudinal de los cambios en el pensamiento de los maestros de los maestros en servicio que completaron un programa de maestría. Entre otros conceptos fundados que surgieron de los datos, "articulación" y "lenguaje local y profesional" fueron dos conceptos que son útiles para definir, al menos en parte, lo que sucede con las creencias de los maestros cuando participan en un programa de capacitación docente. El análisis de datos reveló que el programa de capacitación les dio a los maestros un lenguaje profesional que combinaron con su idioma local para articular o conceptualizar su práctica en el aula. En otras palabras, pudieron acceder a sus propias creencias sobre la enseñanza utilizando el lenguaje profesional del programa.

Se podría argumentar que el éxito de los participantes en el estudio de Freeman (1993) se debió al hecho de que eran maestros en servicio, y que podían aplicar y analizar de inmediato en la práctica el conocimiento obtenido de su formación docente. Sin embargo, Borg (1998) presentó un estudio de caso de un maestro de ESL cuya capacitación previa al servicio tuvo un gran impacto en su enseñanza, a pesar de las experiencias negativas al comienzo de su servicio. Borg postuló que esto podría deberse a una serie de factores, incluida la corta duración de la capacitación inicial del maestro (un programa de cuatro semanas), su enfoque práctico (sesiones de enseñanza práctica todos los días) y la naturaleza reflexiva de la práctica de los capacitadores. Borg también sugirió que los estudios que exploran los procesos mediante los cuales los maestros toman decisiones de enseñanza de gramática pueden tener un impacto mucho mayor en la creación de programas de capacitación de maestros L2 más efectivos. De hecho, hay evidencia que muestra que tener en cuenta las creencias previas de los maestros en formación sobre la enseñanza de la gramática es importante si un programa desea tener algún impacto en estas creencias. Una forma es llevar estas ideas a la superficie para reflexionar. Como afirman Richards, Gallo y Renandya (2001), “el desarrollo profesional que involucra a los 
maestros en una exploración directa de sus creencias y principios puede brindar la oportunidad de una mayor autoconciencia a través de la reflexión y el cuestionamiento crítico como puntos de partida para una adaptación posterior" (p.12).

Farrell (1999) hizo exactamente esto; les pidió a sus maestros en formación que completaran una tarea de reflexión y les pidió que escribieran 1) un relato de su experiencia de aprendizaje de idiomas, 2) un plan de lección de gramática y lo enseñarlo, y 3) reflexiones antes, durante y después de la lección. Descubrió que al reflexionar sobre sus experiencias como estudiantes y como docentes, estos pudieron acceder a sus creencias tácitas, examinarlas y, de hecho, cambiar algunas de ellas, o al menos reconocer su impacto en su práctica.

De hecho, muchos investigadores han visto el valor de la investigación narrativa y reflexiva en el desarrollo profesional de los maestros en servicio y en servicio.

Johnson y Golombek (2011) argumentan que reconstruir sus experiencias a través de la narrativa les permite a los maestros hacer explícitas sus ideas y creencias tácitas y, por lo tanto, tener un mayor sentido de autoeficacia y la capacidad de actuar con previsión.

La investigación sobre el impacto de los programas de capacitación docente en las creencias de los docentes examinados aquí revela que los programas efectivos de capacitación docente brindan a los estudiantes un lenguaje profesional con el cual articular sus ideas y la oportunidad de reflexionar sobre sus creencias no examinadas sobre la enseñanza y analizarlas a la luz de sus nuevos conocimientos. En el estudio que se propone aquí, los participantes escribirán en los diarios de los estudiantes a lo largo de su programa de capacitación docente de un año para sacar a la luz sus creencias no supervisadas sobre la enseñanza de la gramática.

\section{La investigación que explora la relación entre las creencias y la práctica en el aula}

El aspecto final de las creencias de los profesores de idiomas en servicio que se tendrán en cuenta en este estudio es cómo esas creencias se manifiestan (o no) en la práctica en el aula, o en el caso del estudio propuesto aquí, la enseñanza de los estudiantes de los participantes. Como señaló Borg (2003), se debe hacer una distinción entre el cambio de comportamiento y el cambio cognitivo, ya que el primero no garantiza el segundo, y viceversa. Si bien el enfoque de este estudio será examinar el cambio (o su falta) de las creencias de los maestros en servicio sobre la enseñanza de la gramática, no se puede ignorar que el objetivo final de cualquier programa de capacitación de maestros es impactar la práctica de los maestros en el aula y, por lo tanto, También se debe examinar la relación entre sus creencias y la práctica en el aula.

Hay muchos estudios que han demostrado que las creencias expresadas por los docentes no siempre se reflejan en sus prácticas en el aula. En un estudio de caso de dos maestros experimentados, Farrell y Lim (2005) encontraron incidencias de convergencia y divergencia 
entre creencias y prácticas en el aula. Muchas de las razones citadas para la divergencia fueron contextuales, tales como limitaciones de tiempo, requisitos curriculares, demandas de administración escolar y expectativas de los padres. Los investigadores también conjeturaron que las creencias y actitudes inconscientes y arraigadas sobre la efectividad de la instrucción gramatical tradicional también pueden ser un factor, ya que ambos maestros admitieron no ser conscientes de sus creencias sobre la enseñanza de la gramática hasta que se les preguntó.

El estudio de Farrell y Lim (2005) presenta puntos importantes sobre factores que pueden influir en una divergencia entre las creencias y la práctica. Investigaciones previas han demostrado que los factores contextuales, como el ambiente escolar y el comportamiento de los estudiantes tienen un impacto importante en las prácticas en el aula, especialmente en los maestros novatos (Spada y Massey 1992, Pennington y Richards 1997, Richards y Pennington 1998, según lo citado por Borg 2006). Siendo este el caso, es importante tener en cuenta que hay muy pocos estudios que se hayan realizado en contextos que sean más representativos de las aulas de idiomas a escala global, ej. escuelas públicas con maestros no nativos que trabajan con un plan de estudios ordenado por el estado (Borg 2003). El estudio propuesto aquí estará preparado para abordar esta brecha en la investigación, ya que el entorno es uno que refleja este tipo de factores contextuales.

Además, el factor de que los maestros desconocen sus propias creencias es importante. Otros estudios que han examinado las creencias de los maestros (Breen, Hird, Milton, Oliver y Thwaite 2001, por ejemplo) han declarado que los participantes mencionaron que no habían articulado sus creencias antes de participar en dicho estudio. Esto respalda la conjetura de Farrell (2005) de que, para muchos maestros, aunque afirman adherirse a creencias que reflejan el paradigma de enseñanza actual, pueden ser sus creencias no examinadas las que influyen en sus acciones en el aula. Como se mencionó anteriormente, el estudio propuesto aquí tendrá un elemento reflexivo que precederá a la práctica en el aula, lo que con suerte ayudará a los participantes a articular sus creencias tácitas sobre la enseñanza de la gramática.

Otro factor que podría explicar la divergencia de los maestros de sus creencias declaradas sobre la enseñanza de la gramática es la creencia del maestro sobre su propio dominio de la gramática. Un ejemplo de esto es Borg (2005), que exploró la toma de decisiones a través de estudios de caso de maestros en servicio. Borg postuló que un factor importante que impactó la toma de decisiones de los maestros en el aula fue su percepción de su propio conocimiento sobre el lenguaje. Por ejemplo, la falta de confianza de un maestro en su capacidad para explicar una pregunta de gramática improvisada de un alumno tuvo un impacto directo en la forma en que diseñó las actividades de gramática en su clase. 
Phipps y Borg (2009), exploraron más a fondo las "tensiones" entre las creencias gramaticales de los maestros y las prácticas en el aula, utilizando el término de Freeman (2003) para la divergencia entre los dos. En un estudio cualitativo de tres profesores experimentados de ESL en Turquía, se centraron en distinguir entre creencias centrales y periféricas y sus relaciones con la práctica en el aula. Descubrieron que los maestros eran más propensos a tomar decisiones en clase que eran consistentes con sus creencias básicas, que eran más generales y profundas, y estaban más dispuestos a hacer cosas que iban en contra de sus creencias periféricas, que eran más específicas y más fáciles de identificar por los propios profesores. Esta distinción entre creencias centrales y periféricas es importante, ya que va más allá de identificar tensiones y explora las razones que las subyacen.

Como se demostró aquí, hay muchos factores que han demostrado causar divergencia o tensiones entre las creencias de los maestros y su práctica en el aula. Algunos de estos son factores contextuales, conciencia de las propias creencias, creencias sobre el propio conocimiento y la relación entre las creencias centrales y periféricas. Todos estos factores se tendrán en cuenta en las observaciones realizadas en este estudio.

\section{PREGUNTAS DE INVESTIGACIÓN}

El estudio propuesto busca responder las siguientes preguntas de investigación:

1. ¿Cómo (si es que lo hacen) cambian las creencias de los maestros en servicio sobre la enseñanza de la gramática después de completar un programa de licenciatura de cinco años en la enseñanza del idioma inglés y con cierta experiencia acumulativa?

2. ¿Cuál es la relación entre las creencias de los maestros en servicio sobre la enseñanza de la gramática y su práctica en el aula?

\section{METODOLOGÍA}

El presente estudio de métodos mixtos está diseñado para mezclar datos cualitativos y cuantitativos para comprender mejor la problemática estudiada. El propósito es utilizar diferentes estrategias de investigación al recopilar datos para obtener un equilibrio al combinar métodos cuantitativos y cualitativos. Posteriormente, esta decisión proporciona un acercamiento cercano a las fortalezas y debilidades de ambos métodos (Johnson y Turner, 2003).

Los participantes en este estudio fueron 20 profesores de inglés de secundaria en servicio que recibieron una beca patrocinada por la Embajada de los Estados Unidos en Nicaragua. Esta beca consistió en un programa de capacitación de un año (noviembre de 2016 a noviembre de 2017) dividido en cuatro módulos diferentes. Los módulos uno y dos se centraron en el desarrollo de sus habilidades lingüísticas y los módulos 3 y cuatro se dedicaron a capacitar a los maestros en metodología de enseñanza. Todos los participantes tenían una licenciatura en TEFL (enseñanza 
del inglés como lengua extranjera) y su experiencia en la enseñanza del inglés osciló entre 5 y 13 años. Vale la pena mencionar que los maestros de inglés en Nicaragua deben pasar cinco años de estudio para obtener su licenciatura en el programa TEFL. Durante su programa TEFL de cinco años, los estudiantes docentes toman dos clases de metodología de enseñanza. El estudio comenzó en su primer módulo.

Los datos para este estudio se recopilaron utilizando las siguientes herramientas: cuestionarios, diarios y observaciones en el aula. La primera herramienta utilizada fueron los diarios de aprendizaje. El registro en diario es una forma de recopilación de datos introspectivos que sirve como una excelente forma de recopilar datos longitudinales (Bartels, 2005). Los participantes completaron su primer diario de aprendizaje en su primer módulo del programa. Borg (2005) también sugirió que los cursos en un programa de formación de profesores de idiomas deberían entrelazar el conocimiento sobre el lenguaje y la metodología. Al pedirles a los estudiantes que reflexionen sobre sus creencias sobre la enseñanza y el aprendizaje de idiomas como parte de su clase de idiomas, se darán cuenta de sus propias creencias y al mismo tiempo proporcionarán datos para este estudio.

Si bien las indicaciones del diario fueron el proceso de enseñanza-aprendizaje de idiomas en general, las siguientes preguntas se utilizaron para explorar sus creencias sobre la enseñanza de la gramática y sirvieron como datos para este estudio:

1. ¿Cómo les enseñaron gramática sus profesores de inglés de secundaria? ¿Crees que fue efectivo?

2. ¿Cuál crees que es la mejor manera de enseñar gramática? ¿Aprender gramática?

Al final de su cuarto programa de módulo, cuando han alcanzado al menos un nivel de inglés avanzado básico y han tomado sus módulos de métodos de enseñanza, se les pidió a los participantes que respondieran estas preguntas del diario una vez más para comparar cómo o si sus respuestas fueron diferentes.

El segundo instrumento de recopilación de datos aplicado fue un cuestionario sobre sus creencias sobre la enseñanza de la gramática (véase el apéndice A). El cuestionario fue entregado a los participantes al final de su tercer módulo dentro del programa de un año. Para entonces, los profesores de inglés en servicio habrían completado los módulos uno y dos de inglés básico. Se esperaba que la combinación de los diarios de los estudiantes y el cuestionario no solo arrojaría datos sobre sus creencias iniciales de enseñanza de la gramática, sino que también permitiría a los participantes externalizar y, por lo tanto, confrontar y analizar dichas creencias (Johnson y Golombek 2011). Algunas de las declaraciones incluidas en el cuestionario fueron tomadas del Inventario de Creencias de Maestros de Johnson (1992) (según lo citado por Richards y Lockhart 
1996). Las declaraciones se escriben para reflejar un enfoque explícito y deductivo de la enseñanza y el aprendizaje de la gramática (generalmente considerado como la forma tradicional de enseñar gramática) o un enfoque implícito e inductivo (un enfoque más basado en la comunicación). Cada declaración fue calificada en una escala Likert de 5 puntos, con 5 representando un fuerte acuerdo y 1 fuerte desacuerdo. Una limitación en el uso de un cuestionario de este tipo es que es por naturaleza general y sin contexto (Bartels 2005). Además, como con todos los tipos de autoinforme, ni el cuestionario ni los diarios de los alumnos representan los pensamientos reales de los participantes, sino más bien su estimación de sus pensamientos. Sin embargo, al combinar esto con la observación en el aula, estas limitaciones pueden superarse hasta cierto punto.

La etapa final de la recopilación de datos fue la observación en el aula. En el cuarto módulo, los maestros de inglés en servicio debían preparar y presentar planes de lecciones. Estos planes de lecciones se estructuraron utilizando libros de texto y programas de estudios prescritos por el Ministerio de Educación en Nicaragua para brindarles a los participantes la oportunidad de compartir con la clase cómo trabajan en la vida real y evitar prescribir o imponerles estructuras de libros de texto, programas de estudio o planes de lecciones para seguir rígidamente. Se realizarán observaciones del aula no participativa para respaldar los datos principales y ver cómo los participantes presentan la gramática en la clase. Después de cada observación en el aula, la información obtenida se analizará para encontrar cualquier divergencia entre su práctica y sus creencias informadas.

\section{ANÁLISIS Y DISCUSIÓN DE RESULTADOS}

La naturaleza de la recopilación de datos en este estudio requirió un análisis continuo de datos, primero comparando los informes iniciales de las creencias gramaticales con las creencias gramaticales informadas al final del cuarto módulo, y luego una comparación de las creencias informadas con sus prácticas en el aula en el cuarto módulo. El primer paso del análisis de datos fue un análisis estadístico descriptivo de la encuesta. Estos datos se analizaron mediante análisis temático tal como lo definen Braun y Clarke (2006). Estos autores definen el análisis temático como "un método para identificar, analizar e informar patrones (temas) en los datos" (p. 79).

\section{EL CUESTIONARIO}

El cuestionario muestra que los participantes están de acuerdo en que el lenguaje es un conjunto de estructuras gramaticales que necesitan ser aprendidas. Argumentan que una vez que los alumnos dominen ciertas estructuras gramaticales podrán hacer nuevas oraciones. Sin embargo, es interesante observar las respuestas de los participantes en las preguntas 2 y 5 , en las que creen que no es necesario enseñar a los estudiantes cómo usar o aprender diferentes 
reglas gramaticales para hablar bien en un idioma extranjero. Es bastante convincente ver que los participantes creen que la gramática se debe aprender, pero no se debe enseñar.

Las respuestas a las preguntas 6 y 8 indican que los participantes creen que la gramática debe presentarse lo más clara posible durante la instrucción y ven la gramática como un elemento fundamental para hablar con fluidez el idioma. La pregunta 9 refuerza las creencias de los participantes al poner la gramática como un pilar importante entre entender y ser entendido cuando se comunica en inglés. Sin embargo, es visible la contradicción de los participantes en la pregunta 10, en la que una buena mayoría señala la importancia de exponer a los estudiantes al idioma y ven que las explicaciones gramaticales son innecesarias.

Dadas las estadísticas en las preguntas 11 a 14, está fuertemente marcado las creencias de los participantes hacia el aprendizaje y la enseñanza de la gramática. Se estima que el 85\% de los participantes cree que la comunicación no sería posible sin aprender las reglas de gramática y enfatiza que los maestros deberían pasar al menos parte de la instrucción de la clase explicando la gramática.

Sin embargo, existe una contradicción con las respuestas dadas en la pregunta 15, en la que una buena mayoría ve (45\%) la enseñanza de la gramática como innecesaria y hace hincapié en dar a los estudiantes la oportunidad de usar el idioma en situaciones reales y eventualmente hacer que descubran las reglas por sí mismas (ver Apéndice B).

\section{DATOS DEL DIARIO}

Las respuestas de los diarios se analizaron y resumieron de la siguiente manera:

\begin{tabular}{|l|l|}
\hline $\begin{array}{l}\text { ¿Cómo enseñaron gramática tus profe- } \\
\text { sores de inglés de secundaria? ¿Creías } \\
\text { que era efectivo? }\end{array}$ & $\begin{array}{c}\text { ¿Cuál es la mejor manera de enseñar } \\
\text { gramática? ¿Aprender gramática? }\end{array}$ \\
\hline $\begin{array}{l}\text { Los profesores explicaron mucha gramáti- } \\
\text { ca. Escribimos oraciones y las cambiamos } \\
\begin{array}{l}\text { a negativas. Entendí la gramática bastante } \\
\text { bien. }(3)^{1}\end{array}\end{array}$ & $\begin{array}{l}\text { La mejor manera de enseñar gramática es } \\
\text { a través de canciones, comenzando por } \\
\text { estructuras básicas. (2) }\end{array}$ \\
\hline $\begin{array}{l}\text { Los profesores enseñaron gramática en } \\
\text { español cuando explicaron las reglas. Creo } \\
\text { que fue efectivo. (3) }\end{array}$ & $\begin{array}{l}\text { La mejor manera de enseñar gramática es } \\
\text { palabra por palabra, poniendo énfasis en } \\
\text { la estructura. } \\
\text { (3) }\end{array}$ \\
\hline \multicolumn{2}{|c}{ La tabla continúa en página siguiente» } \\
\hline
\end{tabular}

1. Los números mostrados en la tabla indican la frecuencia de las respuestas de los participantes en el diario. 


\begin{tabular}{|l|l|}
\hline $\begin{array}{c}\text { ¿Cómo enseñaron gramática tus profe- } \\
\text { sores de inglés de secundaria? ¿Creías } \\
\text { que era efectivo? }\end{array}$ & $\begin{array}{c}\text { ¿Cuál es la mejor manera de enseñar } \\
\text { gramática? ¿Aprender gramática? }\end{array}$ \\
\hline $\begin{array}{l}\text { Los maestros nos proporcionaron un mo- } \\
\text { delo, luego seguimos el modelo y escribi- } \\
\text { mos muchas oraciones. (5) }\end{array}$ & $\begin{array}{l}\text { La mejor manera de enseñar gramática es } \\
\text { decirles a los estudiantes que investiguen } \\
\text { las reglas gramaticales por adelantado y } \\
\text { que le expliquen a la clase. } \\
(3)\end{array}$ \\
\hline $\begin{array}{l}\text { Algunos maestros explicaron la gramática } \\
\text { comparando español con inglés. Encontré } \\
\text { esta manera muy efectiva.. (7) }\end{array}$ & $\begin{array}{l}\text { La mejor manera de aprender gramática es } \\
\text { conectarlo con ejercicios de vocabulario. } \\
(2)\end{array}$ \\
\hline $\begin{array}{l}\text { No recuerdo cómo los maestros enseñaban } \\
\text { gramática. (2) }\end{array}$ & $\begin{array}{l}\text { La mejor manera de enseñar gramática es } \\
\text { proporcionar a los estudiantes un modelo } \\
\text { a seguir. (6) }\end{array}$ \\
\hline & $\begin{array}{l}\text { La mejor manera de aprender gramática } \\
\text { es memorizar estructuras gramaticales y } \\
\text { practicar diferentes ejercicios en un libro } \\
\text { de gramática. (4) }\end{array}$ \\
\hline
\end{tabular}

Dada la experiencia de los maestros con la gramática en la escuela secundaria, ya sea como maestros o estudiantes, se descubrió que los maestros de inglés de la escuela secundaria favorecían ampliamente la enseñanza de la gramática explícitamente al explicar claramente las reglas y seguir los modelos. Además, algunos de los participantes creen que la enseñanza de la gramática es más efectiva cuando se comparan los dos idiomas y las reglas se explican en español. Sin lugar a dudas, existe una fuerte presencia del método de traducción gramatical en la forma en que algunos de los participantes se formaron en sus escuelas secundarias y sus opiniones se refieren al método de traducción gramatical como una forma efectiva de presentar el idioma. Como Richard - Amato (2003) explicó en su libro "Making it Happens", el enfoque básico del método de traducción gramatical es estudiar las reglas gramaticales del lenguaje y practicar estructuras gramaticales utilizando la traducción a la lengua materna. Por ejemplo, Poldauf (1995: 6) sugirió que "la implementación de CA debe proceder de las similitudes a las diferencias entre los sistemas de lenguaje L1 y L2" (como se cita en Du, 2016, p.20).

Los resultados obtenidos en el diario básicamente muestran que los participantes indican que la mejor manera de enseñar gramática es señalando claramente las reglas y estructuras. Por ejemplo, Stern (1992) afirma que los estudiantes usan la traducción para comparar el idioma de destino con su idioma nativo con el objetivo de encontrar similitudes y diferencias de ambos idiomas y evitar dificultades en el aprendizaje del idioma (como lo cita Trugul, 2003). 
Además, algunos de los participantes expresaron que la mejor manera de aprender gramática es aprender reglas y crear una relación entre vocabularioy gramática. Como se menciona en la metodología, el diario se administró en el primer módulo y luego en el cuarto módulo. El objetivo era proporcionar a los maestros en servicio más oportunidades para reflexionar. Sin embargo, las respuestas no cambiaron al comparar ambas diarios.

\section{OBSERVACIÓN EN EL AULA}

Las observaciones de los 20 participantes que entregaron sus planes de lecciones se resumieron de la siguiente manera: catorce de los participantes enseñaron gramática explicando explícitamente las reglas gramaticales y proporcionando tres o cuatro ejercicios para practicar. Según Larsen - Freeman (2001) en el artículo “Enseñanza de gramática”, explica que "el propósito de la gramática no es solo dar reglas, porque incluso si los alumnos conocieran todas las reglas, esto no garantizaría que pudieran producir ciertos patrones gramaticales cuando se comunican en un contexto particular" (p.5).

Seis de los participantes no incluyeron la gramática en sus planes de lecciones, pero decidieron explicar la gramática al final. Explicaron la gramática escribiendo un ejemplo y explicando el equivalente en español. Es importante mencionar que algunos participantes respondieron en una de las preguntas del diario que encontraron efectivas cuando su maestra de inglés de secundaria explicó las reglas de gramática usando el español. Auerbach (1993) considera que el uso de L1 es positivo y cree que el L1 refuerza la sensación de seguridad de los alumnos cuando se expresan. Es interesante mencionar que el investigador no pidió a los participantes que escribieran sus planes de lecciones basados en la gramática. En cambio, se les pidió a los participantes que diseñaran sus planes de lecciones de la forma en que los escriben en sus escuelas. El objetivo era crear un entorno natural en el que los participantes se sintieran cómodos y no se midieran específicamente en la forma en que presentaban la gramática (véase el apéndice $C$ ).

\section{INTERPRETACIÓN DE RESULTADOS}

Sin lugar a dudas, los resultados muestran que la gramática juega un papel muy importante en el aprendizaje y la enseñanza. El aprendizaje formal de la gramática se ve como un vehículo importante para la comunicación exitosa en inglés en la cual el enfoque deductivo es el enfoque principal. Además, los participantes pusieron mucho énfasis en la enseñanza y el aprendizaje de la gramática al entregar sus planes de lecciones. Es interesante mencionar que pasaron una cantidad considerable de tiempo explicando la gramática en su plan de lección, lo que indicaba de alguna manera que estaban influenciados por sus propias experiencias como aprendices, como se muestra en los resultados obtenidos en el diario, que posteriormente influyeron en sus decisiones al impartir la clase. Sin embargo, no es fácil saber qué guía la decisión o creencia del 
maestro al impartir la clase o al elegir la mejor manera de enseñar o aprender. Según Johnson (1994), comprender qué guía a los docentes en su práctica no es fácil o intentar describir sus creencias tampoco es fácil ni observable como lo cita Abdullah (2012).

Sin embargo, esto parece contradecir la posición de los docentes dada la interpretación de frecuencias y patrones recurrentes en las respuestas a los cuestionarios, donde algunos de ellos apuntalan que no es necesario enseñar o aprender gramática para hablar inglés, cuya posición o creencia no se reflejó realmente en la práctica en el aula. Por ejemplo, Basturkmen (2012), cree que los maestros tienen su propio marco de creencias y, a veces, estas creencias no se reflejan en su práctica docente, pero tienen un profundo impacto en las decisiones de instrucción (según lo citado por Farrel y Bennis, 2013).

Finalmente, los resultados muestran que la creencia de los maestros sobre la enseñanza de la gramática no ha cambiado después de completar su programa TEFL de cinco años y con experiencia acumulada como estudiantes de idiomas y cómo maestros. Por lo tanto, en el presente estudio, los hallazgos sobre el papel de la gramática en el aula están relacionados con otros estudios mencionados en la literatura.

\section{LIMITANTES DEL ESTUDIO}

Una de las limitaciones en el presente estudio fue el pequeño tamaño de la muestra, solo 20 participantes. Hubiera sido interesante administrar una entrevista a los participantes para examinar sus creencias en profundidad. Además, en las preguntas del diario, la segunda pregunta debería haberse dividido en dos: ¿Cuál crees que es la mejor manera de enseñar gramática y cuál crees que es la mejor manera de aprender gramática? Dadas las respuestas, se observó que la mayoría de los participantes centraron sus respuestas en responder "la mejor manera de enseñar gramática en lugar de la mejor manera de aprender gramática”. Este aspecto podría haber aclarado algunas dudas sobre la enseñanza y el aprendizaje de la gramática.

A pesar de las limitaciones mencionadas, el presente estudio pretende representar las creencias de los maestros sobre la enseñanza de la gramática y cómo sus creencias están relacionadas con la práctica en el aula. Cuando se trata de la observación en el aula, quizás no era ético no haberles dicho a los participantes que formaban parte de un estudio de investigación, pero de alguna manera, este aspecto podría haber alertado a los participantes de que estaban siendo observados sobre cómo presentaban la gramática en su clase y, finalmente, los resultados podrían han sido diferentes. Esta decisión se tomó en busca de información objetiva de los participantes. Este estudio no generaliza los resultados, pero pretende arrojar luz sobre las creencias de los maestros sobre la enseñanza de la gramática. 
Se sugiere para futuras investigaciones tener observación en el aula y entrevistas con formadores de docentes a nivel universitario para examinar sus creencias y ver cómo se presenta la enseñanza de la gramática a los docentes de inglés en formación y en servicio. Esto podría aclarar si los maestros de secundaria tienden a replicar cómo aprendieron gramática en su programa TEFL o si sus creencias están influenciadas por otros factores.

\section{CONCLUSIÓN}

Los hallazgos en este estudio intentaron explorar las creencias de los maestros sobre la enseñanza de la gramática en Nicaragua. Los resultados indicaron que existía una inconsistencia entre las creencias de los maestros y la práctica en el aula y sus creencias fueron influenciadas de alguna manera por sus experiencias como estudiantes de secundaria y como profesores en formación también. Todos los maestros fueron una vez estudiantes, y sus creencias sobre la enseñanza son a menudo un reflejo de cómo ellos mismos fueron enseñados. Por lo tanto, se encontró una fuerte presencia de las características del método gramática-traducción en la forma en que los participantes fueron enseñados en inglés mientras eran estudiantes de secundaria. También incluyeron aspectos del método de traducción gramatical al diseñar y entregar sus planes de lecciones. Este estudio no intenta desacreditar a GTM, cuyo enfoque tuvo un éxito notable en el pasado, sino informar sobre las creencias de los maestros sobre la enseñanza de la gramática en Nicaragua.

El estudio también informó que las creencias de los maestros sobre la enseñanza de la gramática parecen no haber cambiado después de cierta experiencia acumulada trabajando como maestros de secundaria y, finalmente, este hecho influye en su práctica docente. Sin embargo, los resultados no pueden generalizarse, pero pretenden representar lo que piensan algunos maestros de secundaria nicaragüenses sobre la enseñanza de la gramática. Finalmente, el estudio proporcionó información importante a los profesores de inglés en general para tomar decisiones con respecto a la enseñanza de la gramática.

\section{BIBLIOGRAFÍA}

Abdullah, M. (2012). Yemeni Teachers’ Beliefs of Grammar Teaching and Classroom
Andrews, S. (2006). The evolution of teachers' language awareness. Language Awareness, 15 (1), 1-19.

Bartels, N. (2005). Applied linguistics and language teacher education. In N. Bartels (Ed.), Researching applied linguistics in language teacher education (1-16). New York, NY: Springer Science+ Business Media, Inc. 
Borg, S. (1998). Teacher's pedagogical systems and grammar teaching: A qualitative study. TESOL Quarterly 32 (1), pp. 9-38.

Borg, S. (1999). Studying teacher cognition in second language grammar teaching. System 27, 19-31.

Borg, S. (2003). Teacher cognition in language teaching: A review of research on what language teachers think, know, believe, and do. Language Teaching, 36, 81-109.

Borg, S. (2005). Experience, knowledge about language and classroom practice in teaching grammar. In N. Bartel (Ed.), Researching applied linguistics in language teacher education (325340). New York, NY: Springer Science+ Business Media, Inc.

Borg, S. (2006). Teacher cognition and language education: Research and practice. London: Continuum. (pp. 75108)

Braun, V. \& Clarke, V. (2006). Using thematic analysis in psychology. Qualitative Research in Psychology, 3, 77-101.

Breen, M. P., Hird, B., Milton, M., Oliver, R., \& Thwaite, A. (2001). Making sense of language teaching: Teachers' principles and classroom practices. Applied Linguistics, 22 (4), 470-501.

Farrell, T. S. C. (1999). The reflective assignment: Unlocking pre-service English teachers' beliefs on grammar teaching. RELC Journal, 30 (1), pp. 1-17.
Farrell, T. S. C., \& Lim, P. C. P. (2005). Conceptions of grammar teaching: A case study of teachers' beliefs and classroom practices. Teaching English as a Second or Foreign Language E-journal, 9 (2), 1-13.

Farrel, T. S. C. \& Bennis, K. (2013). Reflecting on ESL teacher beliefs and classroom practices: A Case Study. RELC Journal, 44 (2) 163-176.

Du, Y. (2016). The Use of First and Second Language in Chinese University EFL Classroom. Springer Science+Business Media Singapore, 15-44.

Johnson, K. E. \& Golombek, P. R. (2011). The transformative power of narrative in second language teacher education. TESOL Quarterly, 45 (3), 486-509.

Johnson, R. B., \& Turner, L. A. (2003). Data collection strategies in mixed methods research. In A.Tashakkori, and C. Teddlie (Eds.), Handbook of mixed methods in social and behavioral research (pp. 297319). Thousand Oaks, CA: Sage.

Larsen-Freeman, D. 2001. Teaching Language: From Grammar to Grammaring. Boston, MA: Heinle \& Heinle.

Phipps, S. \& Borg, S. (2009). Exploring tensions between teachers' grammar teaching beliefs and practices. System, 37, 380-392.

Richard-Amato, P. A. (2003). Making it happen: From interactive to participatory language teaching: theory and practice. White Plains, NY: Pearson Education. 
Richards, J. C., Gallo, P. B., Renandya, W. A. (2001). Exploring teachers' beliefs and the processes of change. PAC Journal, 1 (1), 41-64.

Richards, J. C. \& Lockhart, C. (1996). Reflective teaching in second language classrooms. New York: Cambridge University Press. 29-51.
Tugrul, M. C. (2013). The Grammar Translation Method and the Use of Translation to Facilitate Learning in ESL Classes. Journal of Advances in English Language Teaching 2013; vol. 1, pp. 103105.

\section{APÉNDICE A}

\section{Cuestionario de creencias de enseñanza de gramática}

Este cuestionario es parte de un estudio sobre cómo las creencias de los profesores en formación de inglés sobre la gramática cambian con el tiempo. Responda cada pregunta con cuidado y honestidad. Recuerde, no hay respuestas correctas o incorrectas; lo importante es que indiques tu opinión personal.

Instrucciones: Las siguientes afirmaciones tratan sobre cómo se aprende inglés como lengua extranjera y cómo se debe enseñar. Califique cuan de acuerdo o en desacuerdo está usted con cada afirmación utilizando la escala disponible (5 representa un fuerte acuerdo y 1 representa un fuerte desacuerdo). Puede hacer más comentarios a continuación si lo desea.

\begin{tabular}{|c|c|c|c|c|c|}
\hline & 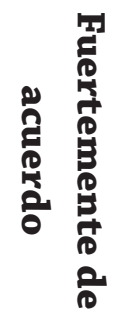 & 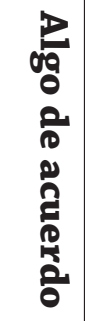 & 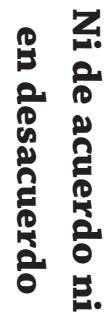 & 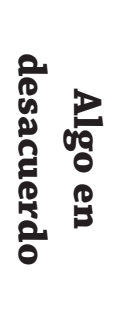 & 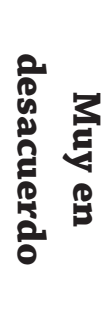 \\
\hline $\begin{array}{l}\text { 1. El lenguaje puede considerarse como un conjunto } \\
\text { de estructuras gramaticales que el alumno aprende } \\
\text { conscientemente y controlada. }\end{array}$ & 5 & 4 & 3 & 4 & 1 \\
\hline $\begin{array}{l}\text { 2. No es necesario enseñar a los estudiantes cómo } \\
\text { usar diferentes reglas gramaticales; Por lo general, } \\
\text { comienzan a hablar inglés por su cuenta. }\end{array}$ & 5 & 4 & 3 & 4 & 1 \\
\hline $\begin{array}{l}\text { 3. El lenguaje puede considerarse una comunicación } \\
\text { significativa y se aprende inconscientemente en } \\
\text { situaciones sociales no académicas. }\end{array}$ & 5 & 4 & 3 & 4 & 1 \\
\hline
\end{tabular}




\begin{tabular}{|c|c|c|c|c|c|}
\hline $\begin{array}{l}\text { 4. Si los estudiantes de inglés entienden algunas de } \\
\text { las reglas gramaticales básicas del idioma, general- } \\
\text { mente pueden crear muchas oraciones nuevas por su } \\
\text { cuenta. }\end{array}$ & 5 & 4 & 3 & 4 & 1 \\
\hline $\begin{array}{l}\text { 5. No es necesario que una persona aprenda cons- } \\
\text { cientemente las reglas gramaticales para comunicarse } \\
\text { bien en un idioma extranjero. }\end{array}$ & 5 & 4 & 3 & 4 & 1 \\
\hline $\begin{array}{l}\text { 6. Es importante proporcionar presentaciones claras, } \\
\text { frecuentes y precisas de las estructuras gramaticales } \\
\text { durante la enseñanza del idioma inglés. }\end{array}$ & 5 & 4 & 3 & 4 & 1 \\
\hline $\begin{array}{l}\text { 7. Si una persona puede escuchar, practicar y re- } \\
\text { cordar ejemplos del idioma que usan los hablantes } \\
\text { nativos, puede aprender el idioma. }\end{array}$ & 5 & 4 & 3 & 4 & 1 \\
\hline $\begin{array}{l}\text { 8. Los estudiantes de inglés generalmente necesitan } \\
\text { entender las reglas gramaticales del inglés para poder } \\
\text { hablar con fluidez. }\end{array}$ & 5 & 4 & 3 & 4 & 1 \\
\hline $\begin{array}{l}\text { 9. Si un estudiante de inglés no conoce las reglas } \\
\text { gramaticales, no podrá entender ni ser entendido } \\
\text { cuando intente comunicarse en inglés. }\end{array}$ & 5 & 4 & 3 & 4 & 1 \\
\hline $\begin{array}{l}\text { 10. Un maestro de inglés simplemente debe tratar } \\
\text { de exponer a los estudiantes al idioma, sin muchas } \\
\text { explicaciones gramaticales. }\end{array}$ & 5 & 4 & 3 & 4 & 1 \\
\hline $\begin{array}{l}\text { 11. Las explicaciones gramaticales en clase ayudan } \\
\text { a los estudiantes a comunicarse en situaciones de la } \\
\text { vida real. }\end{array}$ & 5 & 4 & 3 & 4 & 1 \\
\hline $\begin{array}{l}\text { 12. Un buen profesor de inglés dedica al menos parte } \\
\text { de su clase a explicar las reglas gramaticales. }\end{array}$ & 5 & 4 & 3 & 4 & 1 \\
\hline $\begin{array}{l}\text { 13. Cuando se trata de comunicarse en inglés fuera } \\
\text { del aula, las reglas gramaticales no son útiles. }\end{array}$ & 5 & 4 & 3 & 4 & 1 \\
\hline $\begin{array}{l}\text { 14. Una persona no puede aprender un idioma sin } \\
\text { aprender las reglas gramaticales. }\end{array}$ & 5 & 4 & 3 & 4 & 1 \\
\hline $\begin{array}{l}\text { 15. Un buen profesor de inglés no pierde el tiempo } \\
\text { enseñando reglas gramaticales; él o ella les da a los } \\
\text { estudiantes oportunidades de usar el lenguaje de } \\
\text { manera que refleje la vida real y les permite descubrir } \\
\text { las reglas por su cuenta. }\end{array}$ & 5 & 4 & 3 & 4 & 1 \\
\hline
\end{tabular}




\section{RESULTADOS}

\begin{tabular}{|c|c|c|c|c|c|}
\hline & 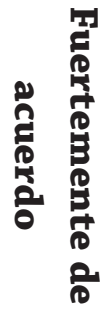 & 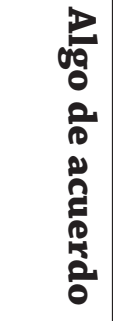 & 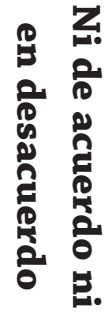 & 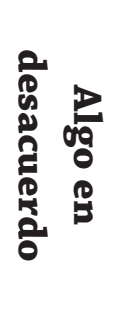 & 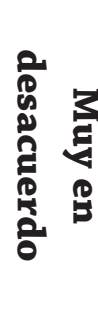 \\
\hline $\begin{array}{l}\text { 1. El lenguaje puede considerarse como un conjunto } \\
\text { de estructuras gramaticales que el alumno aprende } \\
\text { conscientemente y controlada. }\end{array}$ & $\begin{array}{c}7 \\
35 \%\end{array}$ & $\begin{array}{c}5 \\
25 \%\end{array}$ & $\begin{array}{c}5 \\
25 \%\end{array}$ & & $\begin{array}{c}3 \\
15 \%\end{array}$ \\
\hline $\begin{array}{l}\text { 2. No es necesario enseñar a los estudiantes cómo } \\
\text { usar diferentes reglas gramaticales; Por lo general, } \\
\text { comienzan a hablar inglés por su cuenta. }\end{array}$ & $\begin{array}{c}4 \\
20 \%\end{array}$ & $\begin{array}{c}4 \\
20 \%\end{array}$ & $\begin{array}{c}5 \\
25 \%\end{array}$ & & $\begin{array}{c}7 \\
35 \%\end{array}$ \\
\hline $\begin{array}{l}\text { 3. El lenguaje puede considerarse una comunicación } \\
\text { significativa y se aprende inconscientemente en } \\
\text { situaciones sociales no académicas. }\end{array}$ & $\begin{array}{c}6 \\
30 \%\end{array}$ & $\begin{array}{c}5 \\
25 \%\end{array}$ & $\begin{array}{c}5 \\
25 \%\end{array}$ & $\begin{array}{c}2 \\
10 \%\end{array}$ & $\begin{array}{c}2 \\
10 \%\end{array}$ \\
\hline $\begin{array}{l}\text { 4. Si los estudiantes de inglés entienden algunas de } \\
\text { las reglas gramaticales básicas del idioma, general- } \\
\text { mente pueden crear muchas oraciones nuevas por su } \\
\text { cuenta. }\end{array}$ & $\begin{array}{c}14 \\
70 \%\end{array}$ & $\begin{array}{c}6 \\
30 \%\end{array}$ & & & \\
\hline $\begin{array}{l}\text { 5. No es necesario que una persona aprenda cons- } \\
\text { cientemente las reglas gramaticales para comunicarse } \\
\text { bien en un idioma extranjero. }\end{array}$ & $\begin{array}{c}4 \\
20 \%\end{array}$ & $\begin{array}{c}6 \\
30 \%\end{array}$ & $\begin{array}{c}3 \\
15 \%\end{array}$ & $\begin{array}{c}2 \\
10 \%\end{array}$ & $\begin{array}{c}5 \\
25 \%\end{array}$ \\
\hline $\begin{array}{l}\text { 6. Es importante proporcionar presentaciones claras, } \\
\text { frecuentes y precisas de las estructuras gramaticales } \\
\text { durante la enseñanza del idioma inglés. }\end{array}$ & $\begin{array}{c}16 \\
80 \%\end{array}$ & $\begin{array}{c}3 \\
15 \%\end{array}$ & $\begin{array}{c}1 \\
5 \%\end{array}$ & & \\
\hline $\begin{array}{l}\text { 7. Si una persona puede escuchar, practicar y re- } \\
\text { cordar ejemplos del idioma que usan los hablantes } \\
\text { nativos, puede aprender el idioma. }\end{array}$ & $\begin{array}{c}14 \\
70 \%\end{array}$ & $\begin{array}{c}4 \\
20 \%\end{array}$ & $\begin{array}{c}2 \\
10 \%\end{array}$ & & \\
\hline $\begin{array}{l}\text { 8. Los estudiantes de inglés generalmente necesitan } \\
\text { entender las reglas gramaticales del inglés para poder } \\
\text { hablar con fluidez. }\end{array}$ & $\begin{array}{c}11 \\
55 \%\end{array}$ & $\begin{array}{c}3 \\
15 \%\end{array}$ & $\begin{array}{c}3 \\
15 \%\end{array}$ & $\begin{array}{c}1 \\
5 \%\end{array}$ & $\begin{array}{c}2 \\
10 \%\end{array}$ \\
\hline $\begin{array}{l}\text { 9. Si un estudiante de inglés no conoce las reglas } \\
\text { gramaticales, no podrá entender ni ser entendido } \\
\text { cuando intente comunicarse en inglés. }\end{array}$ & $\begin{array}{c}4 \\
20 \%\end{array}$ & $\begin{array}{c}6 \\
30 \%\end{array}$ & $\begin{array}{c}4 \\
20 \%\end{array}$ & $\begin{array}{c}3 \\
15 \%\end{array}$ & $\begin{array}{c}3 \\
15 \%\end{array}$ \\
\hline $\begin{array}{l}\text { 10. Un maestro de inglés simplemente debe tratar } \\
\text { de exponer a los estudiantes al idioma, sin muchas } \\
\text { explicaciones gramaticales. }\end{array}$ & $\begin{array}{c}2 \\
10 \%\end{array}$ & $\begin{array}{c}4 \\
20 \%\end{array}$ & $\begin{array}{c}4 \\
20 \%\end{array}$ & $\begin{array}{c}3 \\
25 \%\end{array}$ & $\begin{array}{c}7 \\
35 \%\end{array}$ \\
\hline
\end{tabular}




\begin{tabular}{|c|c|c|c|c|c|}
\hline $\begin{array}{l}\text { 11. Las explicaciones gramaticales en clase ayudan } \\
\text { a los estudiantes a comunicarse en situaciones de la } \\
\text { vida real. }\end{array}$ & $\begin{array}{c}9 \\
45 \%\end{array}$ & $\begin{array}{c}7 \\
35 \%\end{array}$ & $\begin{array}{c}2 \\
10 \%\end{array}$ & & $\begin{array}{c}2 \\
10 \%\end{array}$ \\
\hline $\begin{array}{l}\text { 12. Un buen profesor de inglés dedica al menos parte } \\
\text { de su clase a explicar las reglas gramaticales. }\end{array}$ & $\begin{array}{c}11 \\
55 \%\end{array}$ & $\begin{array}{c}3 \\
15 \%\end{array}$ & $\begin{array}{c}2 \\
10 \%\end{array}$ & $\begin{array}{c}2 \\
10 \%\end{array}$ & $\begin{array}{c}2 \\
10 \%\end{array}$ \\
\hline $\begin{array}{l}\text { 13. Cuando se trata de comunicarse en inglés fuera } \\
\text { del aula, las reglas gramaticales no son útiles. }\end{array}$ & $\begin{array}{c}2 \\
10 \%\end{array}$ & $\begin{array}{c}5 \\
25 \%\end{array}$ & $\begin{array}{c}4 \\
20 \%\end{array}$ & $\begin{array}{c}1 \\
5 \%\end{array}$ & $\begin{array}{c}8 \\
40 \%\end{array}$ \\
\hline $\begin{array}{l}\text { 14. Una persona no puede aprender un idioma sin } \\
\text { aprender las reglas gramaticales. }\end{array}$ & $\begin{array}{c}5 \\
25 \%\end{array}$ & $\begin{array}{c}8 \\
40 \%\end{array}$ & $\begin{array}{c}1 \\
5 \%\end{array}$ & $\begin{array}{c}2 \\
10 \%\end{array}$ & $\begin{array}{c}4 \\
20 \%\end{array}$ \\
\hline $\begin{array}{l}\text { 15. Un buen profesor de inglés no pierde el tiempo } \\
\text { enseñando reglas gramaticales; él o ella les da a los } \\
\text { estudiantes oportunidades de usar el lenguaje de } \\
\text { manera que refleje la vida real y les permite descubrir } \\
\text { las reglas por su cuenta. }\end{array}$ & $\begin{array}{c}4 \\
20 \%\end{array}$ & $\begin{array}{c}5 \\
25 \%\end{array}$ & $\begin{array}{c}2 \\
10 \%\end{array}$ & $\begin{array}{c}3 \\
15 \%\end{array}$ & $\begin{array}{c}6 \\
30 \%\end{array}$ \\
\hline
\end{tabular}

\section{APÉNDICE B}

\section{Preguntas del diario}

Este diario es parte de un estudio sobre cómo las creencias de los maestros de inglés previos al servicio sobre la gramática cambian con el tiempo. Responda cada pregunta con cuidado y honestidad.

1. ¿Cómo enseñaron gramática tus profesores de inglés de secundaria? ¿Creías que era efectivo?

2. ¿Cuál crees que es la mejor manera de enseñar gramática? ¿Aprender gramática?

\section{APÉNDICE C}

\section{Observación de clase / Reporte}

\section{Introducción:}

En este estudio, he elegido observar a los estudiantes maestros mientras presentan sus planes de lecciones a la clase. El objetivo de esta observación es ver cómo los participantes presentan la gramática en la clase. En los siguientes párrafos, explicaré cómo se realizó la clase y las metodologías de enseñanza del maestro con respecto a la enseñanza de la gramática.

Nota de observación:

Reflexión:

Conclusión: 